\title{
Méthodes de réduction ou d'élimination des risques associés aux tests de la vue en Alberta : recension d'administrations
}

\author{
Alyssa Anderson, candidate \\ à la maîtrise en politiques \\ publiques, B.Sc. \\ Gordon Hensel, O.D. \\ Alberta College \\ of Optometrists
}

\begin{abstract}
Résumé
La réalisation de ce rapport de recherche a été motivée par deux rapports de cas indépendants portant sur des personnes vivant en Alberta, au Canada, qui ont subi une perte de vision permanente en raison de normes de pratique inadéquates entourant les tests de la vue. Les tests de la vue sont habituellement effectués par des opticiens indépendamment des examens oculovisuels complets. Une description des deux rapports de cas et une discussion sur les risques associés aux tests de la vue fournissent des données probantes liées au danger danger pour la santé publique que présentent ces tests. Afin d'étudier les méthodes possibles de réduction ou d'élimination des risques liés aux tests de la vue en Alberta, nous avons effectué une recension des lois et des normes de pratique régissant les tests de la vue au Canada, aux États-Unis, en Nouvelle-Zélande et au Royaume-Uni. Notre recension indique que l'interdiction pure et simple d'effectuer des tests de la vue en Alberta serait le meilleur moyen de protéger l'intérêt public et de réduire les cas évitables de perte de vision. Comme on le constate dans d'autres provinces canadiennes, d'autres approches pour réduire les risques associés aux tests de la vue comprennent 1) l'élaboration et l'application de restrictions concernant l'exécution des tests de la vue et 2) l'élaboration de scénarios clairement définis dans lesquels les opticiens collaborent avec les prescripteurs autorisés pour effectuer des tests de la vue en toute sécurité.
\end{abstract}

MOTS CLÉS :

Test de la vue, examen de la vue, risque, œil, Alberta, santé publique

\section{INTRODUCTION}

Les tests de la vue font intervenir l'utilisation d'équipement de réfraction automatisé afin d'établir les ordonnances de lunettes des patients. Les tests de la vue sont habituellement effectués par des opticiens indépendamment des examens oculovisuels complets. Lorsqu'ils effectuent un test de la vue, les opticiens mesurent l'erreur de réfraction du patient sans toutefois évaluer l'état de sa vision binoculaire ni de sa santé oculovisuelle ${ }^{1}$. En revanche, au cours d'un examen oculovisuel complet, l'optométriste et l'ophtalmologiste évaluent l'état de réfraction, la vision binoculaire et la santé oculaire du patient.

Comme les opticiens n'évaluent pas la vision binoculaire ni la santé oculaire du patient au cours des tests de la vue, des risques sont associés à leur réception :

1) Lorsqu'ils effectuent un test de la vue, les opticiens ne diagnostiquent ni ne détectent les maladies et autres affections de l'œil qui, si elles ne sont pas diagnostiquées et ne sont pas traitées, peuvent entraîner des dommages irréversibles ou une perte de vision permanente. Selon une étude de 2014, plus du quart des patients (26,1\%) âgés de 19 à 64 ans qui se présentent dans une clinique d'optométrie avec des symptômes associés à une réfraction seulement ont reçu aussi un diagnostic de trouble visuel asymptomatique ${ }^{2}$. Cette étude met en évidence la prévalence des maladies oculaires asymptomatiques et souligne le risque associé au découplage du traitement de la réfraction et de l'évaluation de la santé oculaire. 
2) Les patients qui subissent un test de la vue ne connaissent peut-être pas la différence entre un test de la vue et un examen oculovisuel complet et peuvent supposer à tort qu'ils ont reçu une évaluation complète de la santé oculovisuelle ${ }^{3}$. Une étude récente signale un manque général de connaissances du public au sujet des professionnels des soins oculovisuels. L'étude révèle que moins de $25 \%$ des participants des groupes de discussion pouvaient expliquer correctement en quoi se distingue le travail des optométristes par rapport à celui des ophtalmologistes et des opticiens ${ }^{4}$. Si le public ignore la différence entre les professionnels des soins oculovisuels, il est également probable qu'il ne connaisse pas les différents niveaux de soins fournis par chaque profession.

3) Lorsqu'un ophtalmologiste ou un optométriste utilise seulement le résultat d'un test de la vue pour délivrer une ordonnance à un patient (sans vérifier son état binoculaire), le patient est à risque de développer une fatigue oculaire, des maux de tête ou une vision double. Les ordonnances ne devraient être délivrées qu'après examen des résultats de tous les tests effectués au cours d'un examen oculovisuel complet.

\section{RAPPORT DE CAS}

Les rapports de cas suivants portent sur deux personnes vivant en Alberta, au Canada, et constituent des données probantes en lien avec les risques que...risques que comportent les tests de la vue.

Le premier cas concerne une femme de 59 ans qui s'est rendue régulièrement chez un opticien entre 2012 et 2016. Au cours de cette période, l'opticien a effectué plusieurs tests de réfraction sur cette patiente et a transmis les résultats à un ophtalmologiste de l'extérieur qui a prescrit des lunettes après chaque test de la vue. À chaque visite chez l'opticien, la patiente a reçu une ordonnance de lunettes de plus en plus fortes et sa vision s'est détériorée continuellement. Cela a incité la patiente à consulter un optométriste en décembre 2016. Fait à noter, il s'agissait du premier examen oculovisuel complet de la patiente depuis de nombreuses années. L'optométriste a diagnostiqué chez elle un glaucome chronique à angle fermé installé depuis longtemps et, bien qu'un ophtalmologiste de garde ait effectué une intervention chirurgicale d'urgence, la patiente a subi une perte de vision permanente en raison du diagnostic tardif.

Le deuxième cas concerne une jeune fille qui, à l'âge de trois ans, a subi un test de la vue chez un opticien. À l'époque, la mère de l'enfant ne connaissait pas la différence entre un test de la vue et un examen oculovisuel complet. L'opticien ne l'a pas informée de cette importante distinction. Par conséquent, la mère a présumé qu'elle avait fait preuve de diligence raisonnable pour faire examiner les yeux de sa fille. Environ quatre ans plus tard, après avoir subi plusieurs autres tests de la vue, la jeune fille a subi un examen oculovisuel complet chez un optométriste, qui a posé un diagnostic d'amblyopie réfractive anisométropique, ou œil paresseux. L'optométriste s'est associé à un ophtalmologiste pour élaborer un plan de traitement approprié pour l'enfant, lequel exigeait qu'elle porte des lunettes en permanence et qu'elle porte un cache-œil sur son œil sain. Toutefois, en raison du diagnostic tardif, le traitement n'a été que partiellement efficace. La jeune fille n’a jamais retrouvé une vision 6/6 et a subi une perte de vision permanente dans un œil.

Compte tenu de ces deux rapports de cas, il est important de tenir compte des lois et des normes de pratique actuelles concernant les tests de la vue en Alberta. L'annexe 16 de la loi régissant les professions de la santé de l'Alberta accorde aux opticiens inscrits à l'ordre et à l'association des opticiens de l'Alberta (ACAO) le pouvoir légitime d'effectuer des tests de réfraction (tests de la vue) et de déterminer le besoin de porter des lentilles correctrices. Les opticiens effectuant des tests de réfraction en Alberta peuvent administrer des tests de la vue et, après approbation par un prescripteur autorisé, distribuer des lentilles correctrices ${ }^{5}$. Fait à souligner, les normes de pratique de l'ACAO permettent à toute personne, peu importe son âge, ses antécédents médicaux ou la date de son dernier examen oculovisuel complet, d'obtenir un test de la vue. En ce qui concerne l'optométrie, le guide de pratique clinique en télémédecine de l'ordre des optométristes de l'Alberta (ACO) stipule que les optométristes ne doivent pas émettre ni autoriser d'ordonnance optique à partir de tests de réfraction effectués à distance ${ }^{6}$. En ce qui concerne l'ophtalmologie, les normes de pratique en télémédecine de l'ordre des médecins et chirurgiens de l'Alberta (CPSA) stipulent que leurs membres ne peuvent délivrer ou signer une ordonnance, par voie électronique ou autre, à moins d'obtenir les antécédents médicaux et d'effectuer un examen approprié du patient permettant d'établir un diagnostic et de déterminer tout problème sous-jacent ${ }^{7}$. Les rapports de cas décrits ci-dessus démontrent que les lois et les normes de pratique relatives aux tests de la vue effectués par des opticiens en Alberta ne sont peut-être pas adéquates pour protéger l'intérêt supérieur du public. Afin d'étudier les approches visant à réduire les risques liés aux tests de la vue en Alberta, nous avons examiné la réglementation et les normes de pratique en matière de tests de la vue appliquées dans d'autres juridictions. 


\section{RECENSION DE JURIDICTIONS}

La section qui suit présente un résumé de notre recension des lois régissant les tests de la vue au Canada, aux États-Unis, en Nouvelle-Zélande et au Royaume-Uni. Ces juridicitons ont été retenues parce qu'elles réglementent la pratique des opticiens et parce que leur niveau de vie est comparable. Par conséquent, il devrait être plus facile de mettre en œuvre les pratiques de ces juridicitons que celles des juridicitons ayant des niveaux de vie sensiblement différents.

\section{Canada}

Comme le montre le tableau 1, l'Alberta, la Colombie-Britannique et l'Ontario sont actuellement les seules provinces au Canada où les opticiens sont autorisés à effectuer des tests de la vue.

Contrairement à l'ACAO, l'ordre des opticiens de la Colombie-Britannique (COBC) et l'Ordre des opticiens de l'Ontario (OOO) ont imposé des restrictions supplémentaires sur la performance des tests de la vue. Le tableau 2 résume les restrictions en matière d'examen de la vue appliquées en Colombie-Britannique et en Ontario.

En plus des restrictions relatives aux tests de la vue appliquées en Ontario, les normes de pratique de l'Ordre des opticiens de l'Ontario définissent trois scénarios dans lesquels les opticiens et les prescripteurs autorisés peuvent travailler ensemble pour permettre à un opticien d'effectuer des tests de réfraction et de distribuer du matériel optique en toute sécurité. Ces scénarios ont été élaborés après que le gouvernement de l'Ontario a annulé l'autorisation d'exécuter des tests de réfraction de façon autonome qui avait été accordée aux opticiens en 2009. La figure 1 présente chacun des trois scénarios.

Figure 1 : Trois scénarios généraux de l'Ordre des opticiens de l'Ontario en vertu desquels les opticiens peuvent collaborer avec les prescripteurs autorisés pour effectuer des tests de réfraction et distribuer du matériel optique en toute sécurité9.

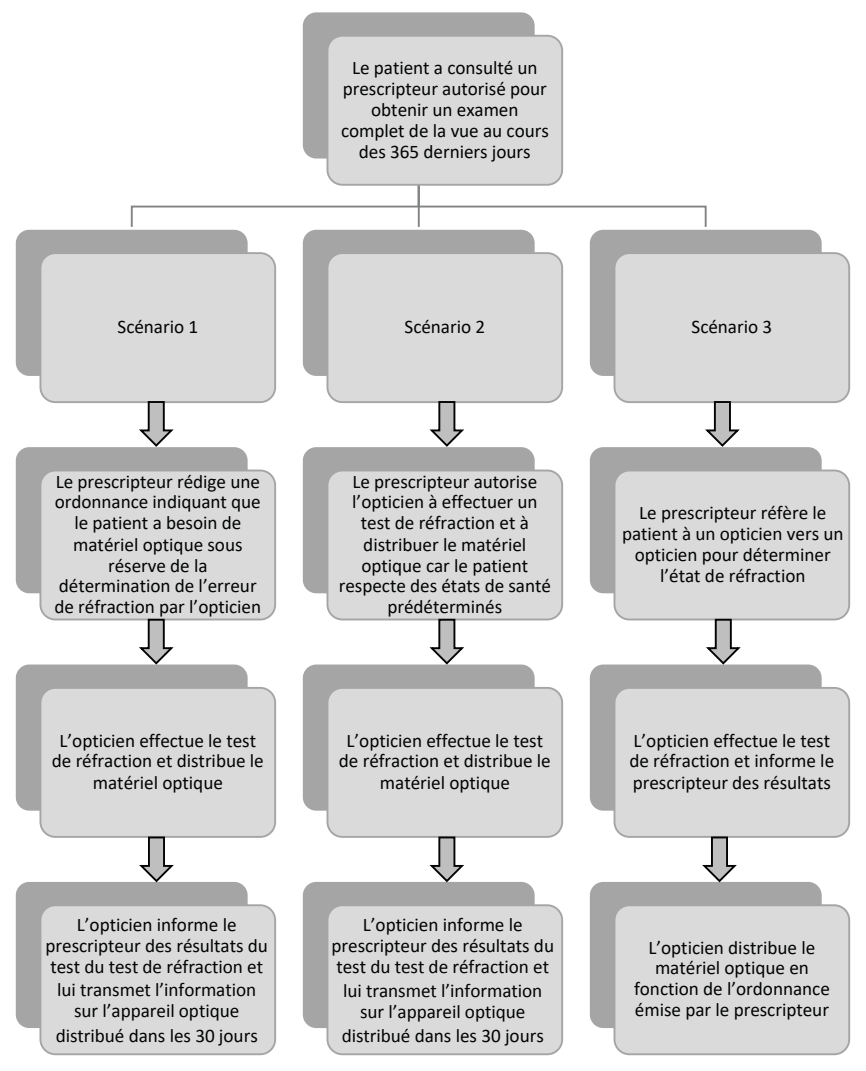

\section{États-Unis}

Aux États-Unis, les 22 États qui réglementent la pratique des opticiens ont adopté des lois qui interdisent explicitement les tests de la vue ou excluent les tests de la vue du champ d'exercice des opticiens (tableau 3). Les 28 États qui ne réglementent pas la pratique des opticiens ont été exclus de cette analyse car ils n'ont pas adopté de loi définissant la pratique des opticiens ni ce qu'elle peut ou ne peut pas inclure. 


\section{Nouvelle-Zélande}

Les opticiens d'ordonnance de la Nouvelle-Zélande ne sont pas autorisés à effectuer les tests de la vue ${ }^{10}$. Le conseil des optométristes et des opticiens d'ordonnances de la Nouvelle-Zélande définit le champ d'exercice des opticiens pour inclure l'interprétation et la délivrance d'ordonnances en matière d'optique et la prestation de conseils et d'instructions sur le matériel optique ${ }^{11}$.

\section{Royaume-Uni}

Au Royaume-Uni, selon l'article 24 de la loi de 1989 régissant la pratique des opticiens, seuls les médecins et optométristes agréés effectuer les tests de la vue ${ }^{12}$. En outre, le General Optical Council, l'organisme de réglementation de la pratique de l'optométrie et de l'optique du Royaume-Uni, a publié une déclaration soulignant qu'aucune partie des examens de la vue ne peut être déléguée à un opticien ou à un opticien de lentilles cornéennes, même sous supervision ${ }^{13}$.

\section{DISCUSSION}

Sur les 34 juridicitons recensées, 31 interdisent aux opticiens d'effectuer des tests de la vue. Par conséquent, à la lumière de cet examen, on peut conclure que l'approche la plus courante et potentiellement la meilleure pour réduire les risques liés aux tests de la vue en Alberta est de les interdire complètement. Lorsqu'il s'agit de protéger et de préserver la santé oculovisuelle, les examens réguliers et complets de la vue constituent la norme de référence puisqu'ils empêchent la perte de vision par le dépistage de maladies asymptomatiques ${ }^{14}$.

Comme l'indique le tableau 1, l'Alberta, la Colombie-Britannique et l'Ontario sont les trois seules provinces du Canada où les opticiens sont autorisés à effectuer des tests de la vue. Fait à noter, le COBC et l'OOO, contrairement à l'ACAO, ont mis en œuvre des restrictions relatives aux tests de la vue concernant l'âge du patient, ses antécédents médicaux, sa vision individuelle, ce qu'il comprend des tests de la vue et la date de son dernier examen de la vue (tableau 2). De plus, l'ACO a récemment fait valoir que les patients qui présentent l'une des situations suivantes ne devraient pas subir de tests de la vue:

1) Avoir moins de 18 ans ou plus de 65 ans

2) Ne pas pouvoir obtenir la meilleure acuité corrigée de 6/12 (20/40) dans l'un ou l'autre des yeux

3) Être atteint de diabète ou de tout autre problème de santé qui peut avoir une incidence sur l'acuité visuelle et causer des changements importants de l'état de réfraction de jour en jour

4) Présenter des antécédents familiaux ou personnels de maladie oculaire

5) Avoir subi un traumatisme oculaire

6) Avoir reçu une ordonnance dont la valeur sphérique est supérieure à $5 \mathrm{D}$

7) Obtenir un changement de cylindre de valeur supérieure à $1 \mathrm{D}$ cylindre supérieure à $1 \mathrm{D}$ par rapport à l'ordonnance précédente

8) Avoir reçu une ordonnance en raison d'un problème de vision binoculaire et/ou une ordonnance qui inclut un prisme

Les restrictions concernant l'âge, les antécédents médicaux ou les problèmes de vision ont une fonction déterminante qui assure que les personnes plus susceptibles d'avoir une maladie oculaire subissent un examen oculovisuel complet plutôt qu'un test de la vue. Par conséquent, les restrictions relatives aux tests de la vue appliquées en Colombie-Britannique et en Ontario et proposées par l'ACO constituent un moyen autre de réduire les risques liés aux tests de la vue en Alberta.

Enfin, les scénarios de collaboration interprofessionnelle de l'OOO offrent une troisième piste de réduction des risques associés aux tests de la vue en Alberta (figure 1). Ces trois scénarios généraux montrent que les opticiens peuvent collaborer avec les prescripteurs dans des situations bien précises pour effectuer des tests de la vue en toute sécurité et distribuer du matériel optique. Pour chaque scénario, il est essentiel que le patient ait subi un examen oculovisuel complet par un optométriste ou un ophtalmologiste au cours de la dernière année. Cette exigence permet d'assurer qu'un professionnel de la santé oculovisuelle formé à la détection des maladies oculaires était sur place pour vérifier les yeux du patient avant qu'un opticien effectue un test de la vue. Si cette exigence avait été mise en place en Alberta, un optométriste ou un ophtalmologiste aurait probablement détecté les problèmes oculaires des deux personnes faisant l'objet des études de cas et l'on aurait pu éviter une issue malheureuse dans les deux cas. À noter qu'en ce qui concerne le deuxième cas, soit l'enfant atteinte d'amblyopie, on ne peut affirmer avec certitude que l'issue malheureuse aurait été évitée si son état avait été détecté et si le plan de traitement avait été lancé plus tôt. Cependant, la recherche montre que les enfants atteints d'amblyopie retrouvent plus d'acuité visuelle avec un traitement précoce qu'avec un traitement entrepris tardivement ${ }^{15}$. Les scénarios de collaboration interprofessionnelle et leur intégration dans les normes de pratique définis par l'ACAO offrent ainsi un autre moyen de s'assurer que les patients reçoivent des soins sécuritaires de la part des opticiens en Alberta. 
Notre étude comprend certaines limites. Tout d'abord, notre article décrit seulement deux cas où la santé de patients a connu une issue malheureuse en raison de normes de pratique inadéquates au sujet des tests de la vue. Il est difficile de mesurer l'ampleur de ce problème dans la province en raison des données manquantes sur le nombre de patients dont les tests de la vue se sont soldés par des résultats malheureux. Deuxièmement, notre recension n’a pas porté sur les 28 États américains ni sur les trois territoires canadiens qui ne réglementent pas la pratique de l'optique. Comme la profession n'est pas réglementée dans ces juridicitons, il a été difficile de déterminer si les opticiens y effectuent couramment des tests de la vue. Pour cette raison, nous avons dû limiter la portée de notre analyse aux juridicitons qui réglementent les opticiens.

\section{CONCLUSION}

Dans ce rapport, nous avons fourni des données probantes relatives aux risques risques pour la santé publique associés aux tests de la vue. D'après notre recension par juridiciton et en raison de la perte de vision permanente citée dans nos deux rapports de cas, nous concluons que le meilleur moyen de réduire les risques associés aux tests de la vue en Alberta est de les interdire complètement. En s'inspirant des normes de pratique mises en œuvre par le COBC et par l'OOO, d'autres approches peuvent comprendre 1) l'élaboration et l'application de restrictions appropriées sur l'exécution des tests de la vue et 2) l'intégration de scénarios de collaboration interprofessionnelle dans des normes de pratique qui définissent clairement la façon dont les opticiens peuvent collaborer avec les prescripteurs autorisés pour effectuer des tests de réfraction et distribuer du matériel optique en toute sécurité.

\section{REMERCIEMENTS}

Nous tenons à remercier les deux personnes dont les rapports de cas figurent dans ce rapport de recherche.

AUTEUR-RESSOURCE

Gordon Hensel, O.D. Courriel : registrar@collegeofoptometrists.ab.ca

Tableau 1 : Aperçu des instances de réglementation et des lois régissant la pratique des opticiens dans les dix provinces du Canada.

\begin{tabular}{|c|c|c|c|}
\hline Juridiciton & Instance de réglementation & Législation applicable & $\begin{array}{l}\text { Les opticiens peuvent- } \\
\text { ils effectuer des tests } \\
\text { de la vue en solo? }\end{array}$ \\
\hline Alberta & $\begin{array}{c}\text { Alberta College and Association } \\
\text { of Opticians }\end{array}$ & Opticians Profession Regulation 45/2011 & Oui \\
\hline $\begin{array}{l}\text { Colombie- } \\
\text { Britannique }\end{array}$ & College of Opticians of B.C & Opticians Regulation 118/2010 & Oui \\
\hline Saskatchewan & $\begin{array}{c}\text { Saskatchewan College of } \\
\text { Opticians }\end{array}$ & The Opticians Act, SS 2010, chap. O-5.1 & Non \\
\hline Manitoba & The Opticians of Manitoba & The Opticians Act, CCSM chap. O60 & Non \\
\hline Ontario & Ordre des opticiens de l'Ontario & Loi de 1991 sur les opticiens, L.O., chap. 34 & Oui \\
\hline Québec & $\begin{array}{l}\text { Ordre des opticiens } \\
\text { d'ordonnance du Québec }\end{array}$ & $\begin{array}{l}\text { Loi sur les opticiens d'ordonnances, RLRQ } \\
\text { chap. O-6 }\end{array}$ & Non \\
\hline $\begin{array}{c}\text { Terre- } \\
\text { NeuveetLabrador }\end{array}$ & $\begin{array}{l}\text { Dispensing Opticians Board of } \\
\text { Newfoundland and Labrador }\end{array}$ & Dispensing Opticians Act, SNL 2005 & Non \\
\hline $\begin{array}{l}\text { Nouveau- } \\
\text { Brunswick }\end{array}$ & $\begin{array}{l}\text { Association des opticiens du } \\
\text { Nouveau-Brunswick }\end{array}$ & Loi sur les opticiens, LN-B 2002, chap. 58 & Non \\
\hline Nouvelle-Écosse & $\begin{array}{l}\text { Nova Scotia College of } \\
\text { Dispensing Opticians }\end{array}$ & $\begin{array}{c}\text { Dispensing Opticians Act, SNS } 2005 \text {, } \\
\text { chap. } 39\end{array}$ & Non \\
\hline $\begin{array}{l}\text { Île-du-Prince- } \\
\text { Édouard }\end{array}$ & $\begin{array}{l}\text { P.E.I Board of Dispensing } \\
\text { Opticians }\end{array}$ & $\begin{array}{c}\text { Dispensing Opticians Act, RSPEI 1988, } \\
\text { chap. D-12 }\end{array}$ & Non \\
\hline
\end{tabular}

Remarque : Les territoires du Canada (Nunavut, Yukon et Territoires du Nord-Ouest) ont été omis de la présente analyse car les opticiens ne sont pas réglementés dans ces juridicitons. 
Tableau 2 : Résumé des restrictions appliquées aux tests de la vue décrites dans les normes de pratique des opticiens de la Colombie-Britannique $e^{8,9}$.

\begin{tabular}{|c|c|c|}
\hline \multirow{2}{*}{$\begin{array}{l}\text { Type de } \\
\text { restriction }\end{array}$} & \multicolumn{2}{|c|}{ Description } \\
\hline & C.-B. & Ontario \\
\hline Âge & $\begin{array}{l}\text { Il est interdit d'effectuer un test de la vue chez les } \\
\text { personnes de moins de } 19 \text { ans ou de plus de } 65 \text { ans. }\end{array}$ & $\begin{array}{l}\text { Il est interdit d'effectuer un test de la vue chez } \\
\text { les personnes de moins de } 19 \text { ans ou de plus de } \\
65 \text { ans. }\end{array}$ \\
\hline $\begin{array}{l}\text { Antécédents } \\
\text { médicaux }\end{array}$ & $\begin{array}{l}\text { Il est interdit d'effectuer un test de la vue chez les } \\
\text { personnes ayant des antécédents individuels ou } \\
\text { familiaux de glaucome, de décollement rétinien, de } \\
\text { dégénérescence maculaire, de diabète ou d'hypertension. }\end{array}$ & $\begin{array}{l}\text { Il est interdit d'effectuer un test de la vue } \\
\text { chez les personnes qui ont des antécédents } \\
\text { individuels de glaucome, de strabisme, de } \\
\text { diabète, de cataractes ou de dégénérescence } \\
\text { maculaire liée à l'âge. }\end{array}$ \\
\hline Vision & $\begin{array}{l}\text { Il est interdit d'effectuer un test de la vue chez les } \\
\text { personnes présentant les conditions suivantes : } \\
\text { 1) Traumatisme crânien ou oculaire survenu au cours } \\
\text { des trois derniers mois } \\
\text { 2) Diplopie } \\
\text { 3) Lentilles correctrices contenant un prisme } \\
\text { 4) Erreur de réfraction dépassant plus ou moins } 6.00 \\
\text { dioptries dans l'un ou l'autre des yeux } \\
\text { 5) Variation de l'erreur de réfraction supérieure à plus } \\
\text { ou moins } 1.00 \text { dioptrie dans l'un ou l'autre des yeux } \\
\text { au cours des } 6 \text { derniers mois ou variation supérieure } \\
\text { à plus ou moins } 2.00 \text { dioptries depuis l'évaluation } \\
\text { enregistrée la plus récente } \\
\text { 6) Meilleure acuité visuelle avec correction de } 6 / 7.5 \\
\text { dans chaque oeil }\end{array}$ & $\begin{array}{l}\text { Il est interdit d'effectuer un test de la vue chez } \\
\text { les personnes suivantes : } \\
\text { 1) Personnes incapables d'obtenir la meilleure } \\
\text { acuité visuelle avec correction de } 6 / 12 \text { dans } \\
\text { chaque œeil } \\
\text { 2) Personnes dont l'évaluation oculovisuelle } \\
\text { préliminaire n'a pas montré une bonne santé } \\
\text { oculovisuelle }\end{array}$ \\
\hline $\begin{array}{c}\text { Compréhension } \\
\text { du patient }\end{array}$ & $\begin{array}{l}\text { L'opticien doit fournir au patient un avis écrit } \\
\text { expliquant la différence entre un examen de la vue } \\
\text { et un examen oculovisuel complet que le patient doit } \\
\text { signer pour indiquer qu'il comprend. }\end{array}$ & $\begin{array}{l}\text { Les patients doivent prendre connaissance des } \\
\text { documents d'information sur la réfraction. }\end{array}$ \\
\hline $\begin{array}{l}\text { Date du } \\
\text { dernier } \\
\text { examen } \\
\text { de la vue }\end{array}$ & $\begin{array}{l}\text { Les personnes de moins de } 40 \text { ans doivent avoir subi un } \\
\text { examen oculovisuel complet depuis qu'elles ont } 19 \text { ans. } \\
\text { Les personnes de plus de } 40 \text { ans doivent avoir subi un } \\
\text { examen oculovisuel complet depuis qu'elles ont } 40 \text { ans. }\end{array}$ & $\begin{array}{l}\text { Il est interdit d'effectuer un test de la vue chez } \\
\text { les personnes qui n'ont pas subi un examen } \\
\text { oculovisuel complet au cours des } 365 \text { derniers } \\
\text { jours. }\end{array}$ \\
\hline
\end{tabular}


Tableau 3 : Aperçu de la législation régissant les tests de la vue effectués par des opticiens dans les 22 États américains qui réglementent la pratique des opticiens.

\begin{tabular}{|c|c|c|}
\hline Juridiciton & Législation applicable & Test de la vue effectué par un opticien \\
\hline Alaska & Lois générales de l’Alaska 08-71 & Interdit en vertu du paragraphe $230(4)$ \\
\hline Arizona & Lois révisées de l'Arizona 321696 & Interdit en vertu du paragraphe $\mathrm{A}(6)$ \\
\hline Arkansas & Code de l'Arkansas 17-89 & Interdit en vertu de l'alinéa 104(c)(1) \\
\hline Californie & $\begin{array}{l}\text { Code des pratiques commerciales et des } \\
\text { professions de la Californie - } 5.4\end{array}$ & Interdit en vertu de l'article 2540 \\
\hline Connecticut & Lois générales du Connecticut 381 & $\begin{array}{l}\text { Test de réfraction exclu de la définition de la pratique des } \\
\text { opticiens traitants en vertu des articles } 20 \text { à } 145\end{array}$ \\
\hline Floride & $\begin{array}{l}\text { Lois générales de la Floride XXXII - } 484.013 \\
\text { Partie I }\end{array}$ & Interdit en vertu du paragraphe $1(3)$ \\
\hline Géorgie & Code officiel annoté de la Géorgie 43-29 & Interdit en vertu de l'alinéa 14(b) \\
\hline Hawaï & Lois générales d'Hawaï - 458 & $\begin{array}{l}\text { Test de réfraction exclu de la définition de la pratique des } \\
\text { opticiens traitants en vertu de l'article } 1\end{array}$ \\
\hline Kentucky & Lois révisées du Kentucky 326 & Interdit en vertu de l'article 326,060 \\
\hline Massachusetts & Règlements du code du Massachusetts 235-2 & $\begin{array}{l}\text { Test de réfraction exclu de la définition de la pratique des } \\
\text { opticiens traitants en vertu de l'article } 2,04\end{array}$ \\
\hline Nevada & Lois révisées du Nevada 637 & Interdit en vertu du paragraphe $637.200(3)$ \\
\hline New Hampshire & Lois révisées du New Hampshire 327-A & $\begin{array}{l}\text { Test de réfraction exclu de la définition d'ordonnance } \\
\text { ophtalmique en vertu de l'article } 1(\mathrm{I})\end{array}$ \\
\hline New Jersey & Lois révisées du New Jersey 5217 & Interdit en vertu de l'article B-41.1 \\
\hline New York & Loi de New York 8-144 & $\begin{array}{l}\text { Test de réfraction exclu de la définition d'ordonnance } \\
\text { ophtalmique en vertu de l'article } 7121\end{array}$ \\
\hline Caroline du Nord & Lois générales de la Caroline du Nord 90-17 & $\begin{array}{l}\text { Test de réfraction exclu de la définition de la pratique des } \\
\text { opticiens traitants en vertu des articles } 90 \text { à } 235\end{array}$ \\
\hline Ohio & Code révisé de l’Ohio 4725 & $\begin{array}{l}\text { Test de réfraction exclu de la définition de la prestation de } \\
\text { services optiques en vertu de l'alinéa } 4725.40 \text { (B) }\end{array}$ \\
\hline Rhode Island & Législation du Rhode Island 535.2 & Interdit en vertu du paragraphe $1(5)$ \\
\hline Caroline du Sud & Code juridique de la Caroline du Sud 40-38 & $\begin{array}{l}\text { Test de réfraction exclu de la définition de la pratique des } \\
\text { opticiens en vertu du paragraphe } 40-38-20 \text { (1) }\end{array}$ \\
\hline Tennessee & Code du Tennessee 63-14 & Interdit en vertu du paragraphe 102(1) \\
\hline Vermont & Lois annotées du Vermont 26-47 & Interdit en vertu du paragraphe $2677(7)$ \\
\hline Virginie & Code de la Virginie 54.1 & Interdit en vertu de l'article 1508 \\
\hline Washington & Code révisé de Washington 18.34 & $\begin{array}{l}\text { Test de réfraction exclu de la définition de la pratique des } \\
\text { opticiens traitants en vertu de l'article } 18.34 .060\end{array}$ \\
\hline
\end{tabular}




\section{RÉFERÉNCES}

1. Canadian Association of Optometrists. Sight Testing Position Statement. Ottawa, ON. 2013. https://opto.ca/sites/default/files/cao_position_statement_-_sight_testing.pdf. Accessed July 2, 2019.

2. Michaud L, Forcier P. Prevalence of asymptomatic ocular conditions in subjects with refractive-based symptoms. J. Optom 2014;7(3):153-160. https://doi.org/10.1016/j.optom.2013.08.003.

3. World Council of Optometry. The Sight Test: Refraction and Examinations of the Eye for the Purpose of Detecting Injury, Disease or Abnormality: The Public Health Case. St. Louis, MO. 2012. https://i2.wp.com/worldcouncilofoptometry.info/wpcontent/ uploads/2017/03/stand_alone_refraction_position_paper_-_approved_oct_2012.jpg?ssl=1. Accessed July 4, 2019.

4. Irving EL, Sivak AM, Spafford MM. "I can see fine": Patient knowledge of eye care. Ophthalmic Physiol Opt 2018;38:422-43. https:// doi.org/10.1111/opo.12566

5. Alberta College and Association of Opticians. Standards of Practice. Edmonton, AB. 2019. https://acao.ca/standards-of-practice-interim/. Accessed July 4, 2019

6. Alberta College of Optometrists. Evidence-Based Clinical Practice Guideline: Telehealth. Edmonton, A.B. Revised June 2019. http:// www.collegeofoptometrists.ab.ca/PDF/Telehealth.pdf. Accessed February 21, 2020

7. College of Physicians \& Surgeons of Alberta. Standard of Practice: Telemedicine. Edmonton, A.B. Revised June 2014. http://www.cpsa. ca/standardspractice/telemedicine/. Accessed February 21, 2020.

8. College of Opticians of British Columbia. Standards of Practice \& Guidelines. Vancouver, B.C. 2011. http://www.cobc.ca/my_folders/Resources/Standards_of_Practice_vl4_FINAL.pdf. Accessed July 10, 2019.
9. College of Opticians of Ontario. Professional Standards of Practice for Opticians in the Province of Ontario. Toronto, ON. 2016. http:// www.coptont.org/docs/Legislation/Standards_of_Practice.pdf. Accessed July 4, 2019.

10. International Opticians Association. "Spotlight on...New Zealand". December 1, 2017. https://www.ioassn.org/news/spotlight-on-newzealand/. Accessed February 21, 2020.

11. Pine L. Notice of Scopes of Practice and Related Qualifications Prescribed by the Optometrists and Dispensing Opticians Board. New Zealand Gazette. 2017. https://gazette.govt.nz/notice/id/2017gs1059. Accessed July 10, 2019.

12. General Optical Council. Opticians Act 1989 Part IV Section 24 https://www.optical.org/en/about_us/legislation/opticians_act.cfm. Accessed September 16, 2019.

13. General Optical Council. Statement on Testing of Sight. 2013 https://www.optical.org/en/news_publications/news_item.cfm/ statement-on-testing-of-sight. Accessed July 10, 2019.

14. Picone G, Brown D, Sloan F, et al. Do routine eye exams improve vision? Int J Health Care Finance Econ 2004;4(1): 43-63. doi:10.1023/ B:IHFE.0000019262.27436.3d

15. Williams C, Northstone K, Harrad RA, et al. Amblyopia treatment outcomes after screening before or at age 3 years: Follow up from randomised trial. BMJ 2002;324(7353):1549. https://doi. org/10.1136/bmj.324.7353.1549 